Approved for public release; distribution is unlimited.
Title:

A NEW PARADIGM FOR 3D COLLISIONLESS MAGNETIC RECONNECTION

Author(s): Giovanni Lapenta

Submitted: Adelaide

July 21-25, 2002

\section{Plasma Theory Group \\ Los Alamos National Laboratory Los Alamos, New Mexico 87545}

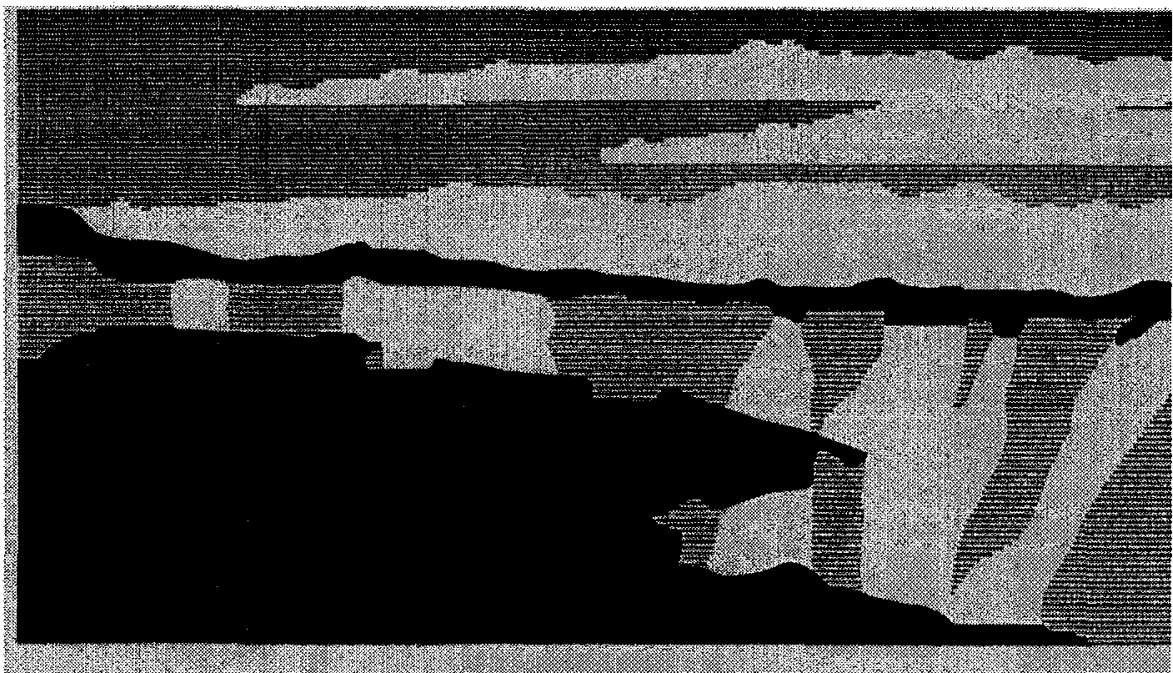

Los Alamos National Labcratory, an affirmative action/equal opportunity employer, is operated by the University of California for the U.S. Department of Energy under contract W-7405-ENG-36. By acceptance of this article, the publisher recognizes that the U.S. Government rete $\equiv$ nonexclusive, royalty-tree llcense to publish or reproduce the published form of this contribution, or to allow others to do so, for U.S. Government purposes. Los A D. National Laboratory requests that the publisher identify this article as work performed under the auspices of the U.S. Department of Energy. The Los Alamos National Laboratory strongly supports academic freedom and a researcher's right to publish; as an institution, however, the Laboratory does not endorse the viewpoint of a publication or guarantee it's technical correctness. 


\title{
A new paradigm for 3D Collisionless Magnetic Reconnection
}

Giovanni Lapenta

Los Alamos National Laboraory, Los Alamos, NM 87545, USA

\begin{abstract}
A new paradigm is emerging for 3D magnetic reconnection where the interaction of reconnection processes with current aligned instabilities plays an important role. According to the new paradigm, the initial equilibrium is rendered unstable by current aligned instabilities (lower-hybrid drift instability first, driftkink instability later) and the non-rniform development of kinking modes leads to a compression of magnetic field lines in certain locations and a rarefaction in others. The areas where the flow is compressional are subjected to a driven reconnection process on the time scale of the driving mechanism (the kink mode). In the present paper we illustrate this series of event with a selection of simulation results.
\end{abstract}

Keywords: sample, $\mathrm{LTEX}_{\mathrm{E}}$

\section{Introduction}

A new paradigm is dawning for 3D magnetic reconnection where the interaction of reconnection processes with current aligned instabilities plays an important role. The new paradigm emerges in kinetic models when the lower-hybrid drift instability (LHDI) and kink modes (KM) (Lapenta and Brackbill, 2002; Daughton, 2002) drive field lines together and promote the onset of reconnection (Lapenta and Brackbill, 2000 ). The new paradigm emerges in MHD models when the presence of velocity shear induces a Kelvin-Helmholtz instability (KHI) that drives reconnection by locally compressing field lines (Brackbill, 19913; Brackbill and Knoll, 2001; Knoll and Brackbill, 2002; Knoll and Chacon, 2002; Lapenta and Knoll, 2002).

According to the new paradigm, reconnection in $3 \mathrm{D}$ is eminently a driven process. The driving force is determined by the instabilities developing in the current aligned direction. For the typical magnetotail configuration with the current in the dawn dusk direction $(y)$ and the field mostly in the tailward direction $(x)$ and the gradients mostly in the north-south direction $(z)$, the tearing instability develops in the $(x, z)$ plane. The collisionless tearing instability has long been considered the best hope to explain reconnection onset. However, accurate studies have not yet resolved the issue of the instability of the tearing mode in actual realistic magnetotail configurations. Most of the results seem to conclude that the tearing mode is stable in realistic configurations and that reconnection onset has other causes (Quest et al., 1996).

(c) 2002 Kluuer Academic Publishers. Printed in the Netherlands.

lapenta.tex; 28/06/2002; 9:46; p.1 
The new paradigm for $3 \mathrm{D}$ reconnection cited above deals with the issue of reconnection onset. It is a new explanation of how reconnection can start, of what mechanism breaks the frozen in condition in the first place. In a spatially varying magnetic field configuration (such as the magnetotail (Lapenta and Brackbill, 2002) or a corona arcade (Lapenta and Knoll, 2002)) or in presence of spatially varying flow shear (such as at different latitudes along the magnetopause (Brackbill and Knoll, 2001; Knoll and Brackbill, 2002)) the non-uniform development of kinking modes leads to a compression of magnetic field lines in certain locations and a rarefaction in others. The areas where the flow is compressional are subjected to a driven reconnection process on the time scale of the driving mechanism (the kink mode). In the new paradigm, two main physics processes must be considered.

First, the kink modes, including both the KHI and the drift kink instability (DKI) (Lapenta and Brackbill, 1997; Daughton, 1999), can drive field lines together, causing a localized compression that drives field lines to reconnect (Brackbill and Knoll, 2001).

Second, new oblique modes are excited and contribute to the process of reconnection (Lapenta and Brackbill, 2000).

This new paradigm is distinct but complementary to the other recent remarkable progress in understanding fast reconnection through the role played by the Hall physics (Biskamp, 2000). The Hall term fast reconnection that has attracted so much recent attention is relevant to the fully developed reconnection process in the non-linear phase. The progress in that area has brought about understanding of the fast reconnection rates observed naturally. But still leaves us at a loss in trying to understand reconnection onset. It is the new paradigm for the role of current aligned instabilities that gives us the tools to understand onset of reconnection.

\section{Physical System}

The Earth's magnetotail is described as usual with a modified Harris equilibrium. The equilibrium is described by the following particle distribution function for species $s$

$$
f_{0 s}=n(z)\left(\frac{m_{s}}{2 \pi k T_{s}}\right)^{3 / 2} \cdot \exp \left[-\frac{m_{s}}{2 k T_{s}}\left(v_{x}^{2}+\left(v_{y}-u_{s}\right)^{2}+v_{z}^{2}\right)\right]
$$

where $u_{s}$ is the drift velocity and $v_{t h, s}=\sqrt{k T_{s} / m_{s}}$ is the thermal velocity of species $s$. The ratio of the ion drift velocity to ion thermal velocity is related to the current sheet thickness $L: u_{i} / v_{t h, i}=2 \rho_{i} / L$ 
where $\rho_{i}$ is the ion gyroradius. The Alfven time is defined as $\tau_{A}=L / v_{A}$ where $v_{A}$ is the Alfven speed.

The usual magnetotail reference frame is used with $x$-axis in the tail direction, $y$-axis in the dawn to dusk direction and $z$-axis along the Earth's polar axis (neglecting the tilt in the Earth's magnetic axis). The initial current $\mathrm{J}_{0}$ is aligned with the $y$ axis. Gradients of the current and magnetic field are aligned with the $z$ axis. The initial magnetic field $\mathrm{B}_{0}$ has a dominant component aligned with the $x$ axis:

$$
B_{x}(x, z)=-B_{o} \mathcal{F}(x) \tanh (\mathcal{F}(x) z / L)
$$

and a vertical component:

$$
B_{z}(x, z)=B_{0} L \mathcal{F}(x) \mathcal{F}^{\prime}(x) \operatorname{sech}(\mathcal{F}(x) z / L)
$$

The variation of the field along the tail is determined by the function $\mathcal{F}(x)$. Two profiles $\mathcal{F}(x)$ are considered here. First, Birn's empirical profile chosen to best fit the actual magnetotail:

$$
\mathcal{F}(x)=[1+(\epsilon / \nu)(x / L)]
$$

where $\nu=0.6$ and $\epsilon$ can be used to vary the ratio of the vertical and horizontal magnetic field component. Second, the quasi-parabolic profile:

$$
\mathcal{F}(x)=\exp (-\epsilon x / L)
$$

The equilibrium charge density is:

$$
n(x, z)=n_{s} \frac{\mathcal{F}^{2}(x)}{\cosh ^{2}(\mathcal{F}(x) z / L)}
$$

To avoid any confusion with velocity-gradient driven modes, no background plasma is added.

The most general perturbation of the initial profile described above can be expressed as:

$$
\psi(x, y, z, t)=\tilde{\psi}(z) \exp \left(i k_{x} x+i k_{y} y-i \omega t\right)
$$

where $\psi$ is the generic field component and $\mathbf{k}=\left(k_{x}, k_{y}\right)$ is the direction of propagation in the equatorial plane. Many different modes have been identified in the magnetotail configuration. The most relevant for the present discussion are the tearing modes (Biskamp, 2000) with $k_{x} \neq 0$ and $k_{y}=0$, the kink modes (Lapenta and Brackbill, 1997; Lapenta and Brackbill, 2002; Daughton, 1999) with $k_{x}=0$ and $k_{y} \neq 0$ and the oblique modes (Lapenta and Brackbill, 2000) that propagate at a non zero angle with both the $x$ and the $y$ axis: $k_{x} \neq 0$ and $k_{y} \neq 0$. 
The system described above evolves according to the Vlasov-Maxwell system. In the present work, relativistic effects will be neglected. The Vlasov-Maxwell system is simulated using the CELESTE 3D implicit particle in cell code. A detailed description of the implicit moment method used in CELESTE 3D can be found in the review paper (Brackbill and Forslund, 1985) and the details of the implementation can be found in ref. (Ricci et al., 2002).

For comparison, we will also consider simulations conducted with a resistive MHD code. The numerical calculations presented here have been obtained using the 3D version of FLIP-MHD (Brackbill, 1991).

\section{Velocity Shears}

The initial equilibrium considered above is unstable to a number of current aligned instabilities, propagating along $y$. Two are of particular importance for understanding reconnection onset: the lower hybrid drift instability (LHDI) and kinking modes (KM).

Recent simulation work (Lapenta and Brackbill, 2002; Daughton, 2002) has shown that the early dynamic of current sheet is dominated by the LHDI. A new effect has been observed and quantified (Lapenta and Brackbill, 2002): the formation of a $y$ independent velocity shear. The nonlinear evolution of the LHDI changes the initial density and current profile and modifies the initial flat velocity profile by creating a velocity shear. Figure 1 shows the velocity profile after saturation of the LHDI. The creation of a robust velocity shear is evident. It sould also be noted that the LHDI growth rate and saturation level is directly proportional to the $\sqrt{T i / T e}$ just like the velocity shear observed in Fig. 1. A further proof that the velocity shear is caused by the LHDI.

It is interesting to note that such process is reminiscent of the zonal flow creation (Drake et al., 1992) observed in tokamak in relation with the L-H transition (Diamond et al., 2000).

Once a velocity shear is created in the short time scales of the LHDI a much slower fluid instability arises, the Kelvin-Helmholtz instability (KHI). The process can be modeled very simply using MHD models. Figure 2-a shows a fully kinetic simulation where the velocity shear is created initially by the LHDI and is destabilized by the KHI. At the end of the simulation the distinctive kinking caused by the KHI is observed. Figure 2-b shows a simple MHD simulation with the same initial equilibrium used for the kinetic simulation but with the addition of an initial velocity shear equal to the velocity shear formed naturally by the LHDI in the kinetic simulation. Clearly, the comparison of Fig. 2-a and Fig. 2-b proves that the evolution following the creation of the shears is 


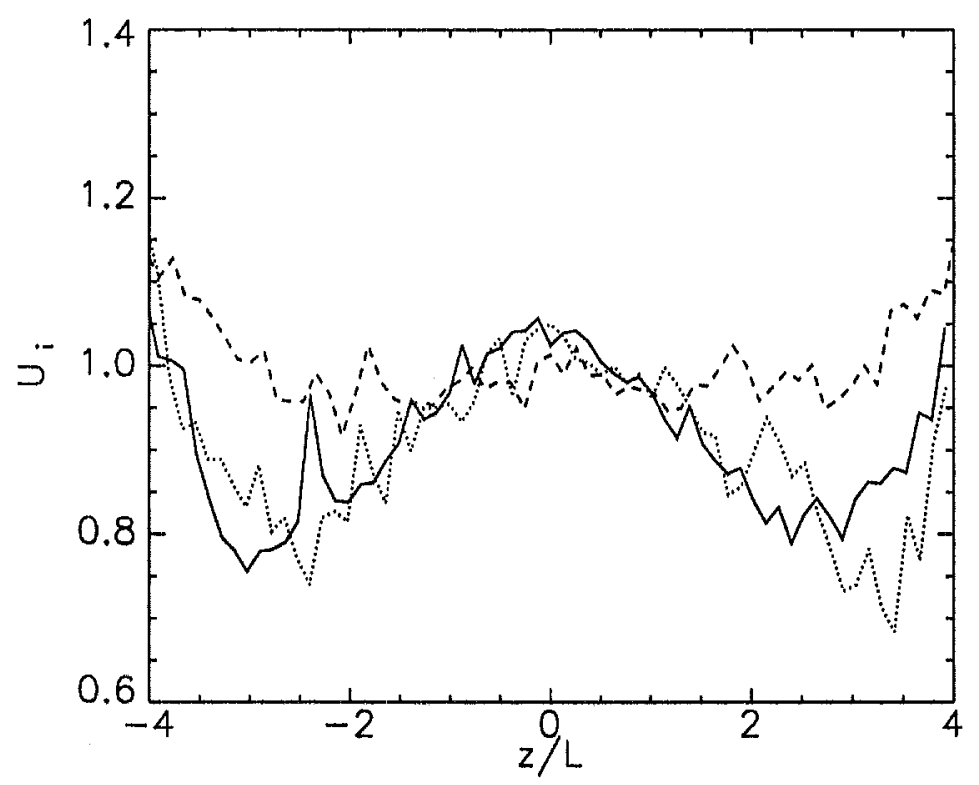

Figure 1. Velocity shear produces by the LHDI in a kinetic simulation. The velocity profle is shown at time $t / \tau_{A}=12$ for a system with $u_{i} / v_{i}=1, m_{i} / m_{e}=180$. Three different temperature ratios are considered: $T_{i} / T_{e}=10$ (solid); $T_{i} / T_{e}=4$ (dotted); $T_{i} / T_{e}=2$ (dashed).

purely a fluid instability. However, note that in the kinetic simulation the velocity shears arise naturally while in the MHD simulation the shears are artificially introduced as an initial condition.

\section{Reconnection Onset}

The evolution of the KHI and the kinking of the initial current sheet has an important consequence on reconnection onset. The presence of kinking causes the compression of the field lines in some regions and the rarefaction in others. Compression of field lines can drive reconnection. Such mechanism has already been observed in MHD simulation of the evolution of the KHI and tearing instability (Brackbill, 19913; Knoll and Chacon, 2002) for application to the Earth magnetopause (Brackbill and Knoll, 2001; Knoll and Brackbill, 2002) and to the solar corona (Lapenta and Knoll, 2002). The present paper is the first instance where the same mechanism is observed in the magnetotail. 
a) kinetic

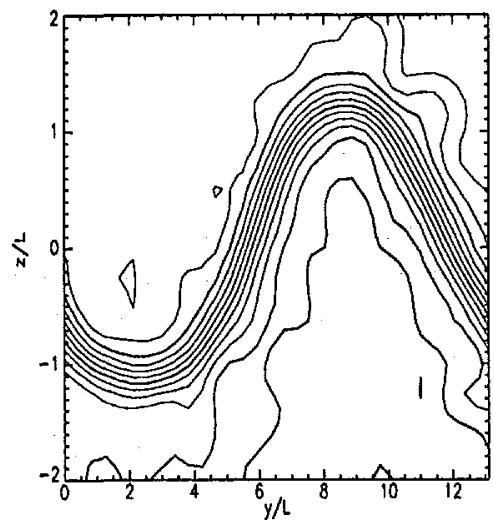

b) resistive $\mathrm{MHD}$

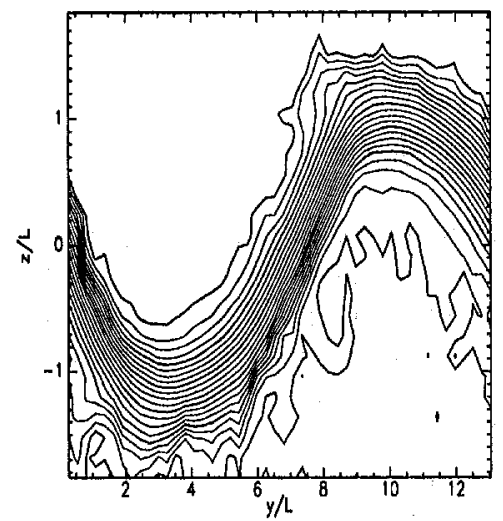

Figure 2. Contour plot of $B_{x}(y, z)$ at the end of two simulation $\left(t / \tau_{A}=110\right)$ : a) a fully kinetic simulation with no initial shear; b) a resistive MHD with an initial shear equal to the shear observed in the kinetic simulation after saturation of teh LHDI. The case considered has $m_{i} / m_{e}=180, u_{i} / v_{t h, i}=1$ and $T_{i} / T_{e}=2$.

In the references just cited, the mechanism was proposed in presence of externally driven shears (driven by the solar wind in the case of the magnetopause (Brackbill and Knoll, 2001) or driven by the photosphere in the case of the corona (Lapenta and Knoll, 2002)). In the magnetotail we suggest that, instead, the shear is created naturally by the LHDI without requiring any external action. Once the current sheet is thin enough for the LHDI to cause the velocity shear the chain of events sets in place and causes the onset of reconnection. The only required external action is the transfer of flux from the dayside to cause the thinning of the magnetotail.

An illustration of how the KHI can drive reconnection onset is presented in Fig. 3. A typical magnetotail (Fig. 3-a) described by eq. (4) is initially unstable to the KHI due to the velocity shears induced by the LHDI. The KHI progresses and causes the compression of certain field lines (Fig. 3-b) eventually leading to driven reconnection (Fig. 3-c).

\section{Conclusions}

A new scenario for magnetotail reconnection has been presented. The scenario is based on a sequence of three events. 


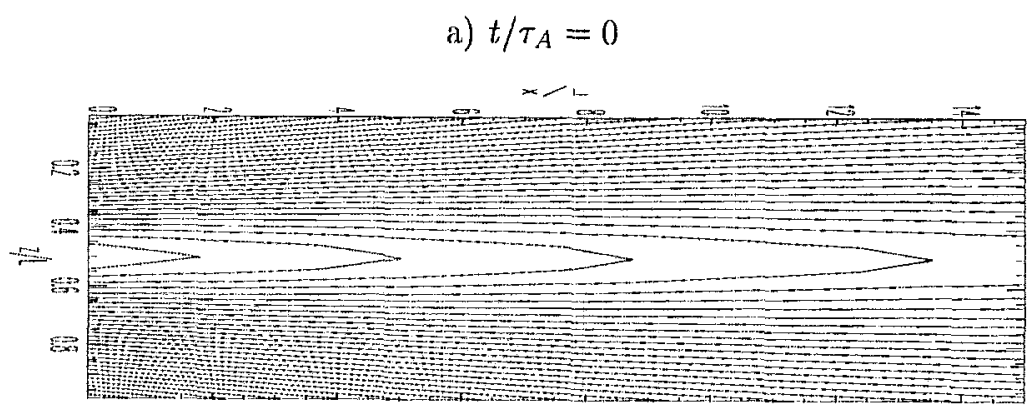

b) $t / \tau_{A}=4$

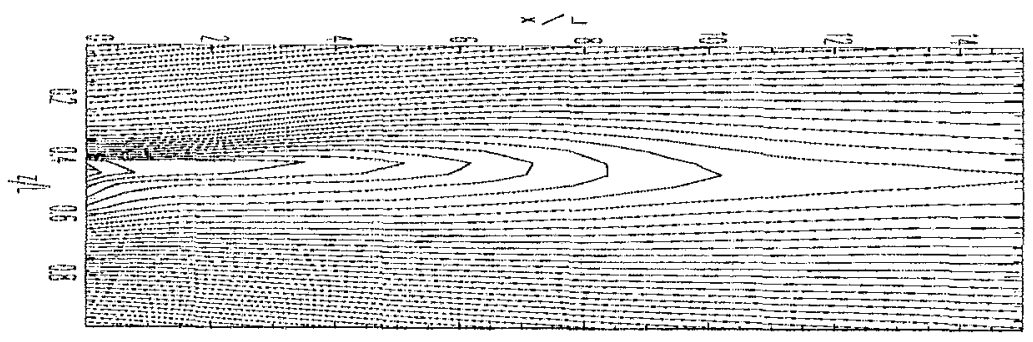

c) $t / \tau_{A}=8$

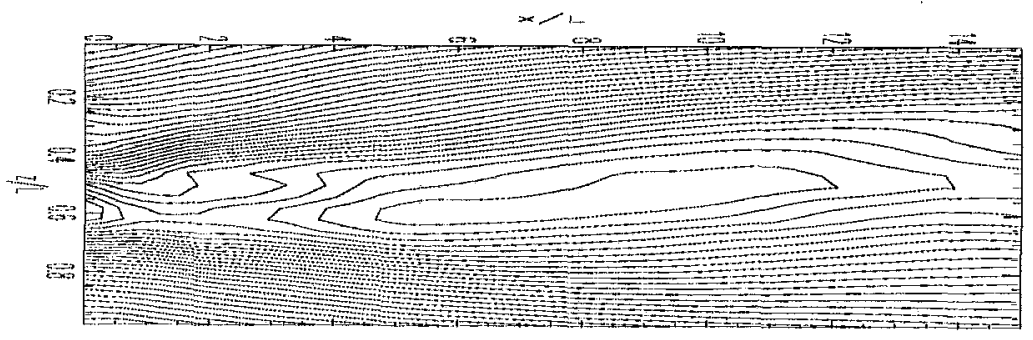

Figure 3. Cross section of the flux surfaces for a 3D MHD simulation at $y=L_{y} / 2$ and three different times: initial $\left(t / \tau_{A}=0\right), t / \tau_{A}=4, t / \tau_{A}=8$.

First, the LHDI grows and saturates changing the initial equilibrium. A consequence of this change is the creation of a velocity shear that renders the equilibrium unstable to the KHI.

Second, the KHT grows and kinks the current sheet causing regions of compression of field lines.

Third, field line compression causes the onset of reconnection. Once reconnection is started by the driving force of the KHI it can progress 
via the action of the Hall term mediated fast reconnection process of recent discovery (Biskamp, 2000).

\section{Acknowledgements}

This research is supported by the United States Department of Energy, under Contract No. W-7405-ENG-36 and by NASA, under the "Sun Earth Connection Theory Program". The supercomputer used in this investigation was provided by funding from JPL Institutional Computing and Information Services and the NASA Offices of Space Science and Earth Science.

\section{References}

Biskamp, D., Magnetic Reconnection in Plasmas (Cambridge University Press, 2000).

Brackbill, J. U., D. W. Forslund, Simulation of Low-Frequency, Electromagnetic Phenomena in Plasmas, in Multiple Time Scales, J. U. Brackbill and B. I. Cohen Eds. (Academic Press, Orlando, 1985), pp. 271-310.

Brackbill, J.U., J. Computat. Phys., 96, 163 (1991).

Brackbill, J.U., J. Computat. Phys., 108, 38 (1993).

Brackbill, J.U., Knoll, D.A., Phys. Rev. Lett., 86, 2329 (2001)

Daughton, W.S., Phys. Plasmas, 6, 1329-1343 (1999).

Daughton, W.S., Phys. Plasmas, submitted (2002).

Diamond P.H., M.N. Rosenbluth, E. Sanchez, C. Hidalgo, B. VanMilligen, T. Estrada, B. Branas, M. Hirsch, H.J. Hartfuss, B.A. Carreras, Phys. Rev. Lett., 84, $4842(2000)$.

Drake, J.F., J.M. Finn, P. Guzdar, V. Shapiro, V. Shevchenko, Phys. Fluids $B, 4$, 488 (1992).

Knoll, D.A., Brackbill, J.U., Phys. Plasmas, submitted (2002).

Knoll, D.A., Chacon L., Phys. Rev. Lett., 8821, 5003 (2002).

Lapenta, G., J.U. Brackbill, J. Geophys. Res., 102, 27099 (1997).

Lapenta, G., J.U. Brackbill, Nonlinear Processes Geophys., 7, 151 (2000).

Lapenta, G., J.U. Brackbill, Physics Plasmas, 9, 1544 (2002).

Lapenta, G., D.A. Knoll, Solar Phys., submitted (2002).

Pritchett, P. L., F. V. Coroniti, J. Geomagn. Geoelectr., 48, 833 (1996).

Quest, K. B., H. Karimabadi, and M. Brittnacher, J. Geophys. Res., 101, 179, (1996).

Ricci, P., G. Lapenta, J.U. Brackbill, J. Computat. Phys., submitted (2002). 